\title{
Analytic Solution for Double Optical Metasurface Beam Scanners
}

Jingru Wang

Huaqiao University

Yuehe Ge ( $\nabla$ yuehe@ieee.org )

Fuzhou University

Zhizhang (David) Chen

Dalhousie University

Zhimeng Xu

Fuzhou University

Hai Zhang

Huaqiao University

\section{Research Article}

Keywords: Analytic Solution, Double Optical Metasurface, Beam Scanners, novel functionalities, inverse solutions, global optimization algorithms, laser scanners

Posted Date: November 30th, 2021

DOI: https://doi.org/10.21203/rs.3.rs-1106825/v1

License: (c) (i) This work is licensed under a Creative Commons Attribution 4.0 International License. Read Full License

Version of Record: A version of this preprint was published at Scientific Reports on April 8th, 2022. See the published version at https://doi.org/10.1038/s41598-022-09877-4. 


\title{
Analytic Solution for Double Optical Metasurface Beam Scanners
}

\author{
Jingru Wang, ${ }^{1}$ Yuehe $\mathrm{Ge}^{*}, 2$ and, Zhizhang (David) Chen ${ }^{2}, 3$, Zhimeng $\mathrm{Xu}^{2}$, and \\ Hai Zhang ${ }^{1}$
}

${ }^{1}$ College of Information Science and Engineering, Huaqiao University, Xiamen 361001, China

${ }^{2}$ College of Physics and Information Engineering, Fuzhou University, Fuzhou, Fujian Province 350108, China

${ }^{3}$ Department of Electrical and Computer Engineering, Dalhousie University, Halifax, Canada

*corresponding. yuehe@ieee.org .

\begin{abstract}
Optical metasurfaces are researched more and more intensively for the possible realization of lightweight and compact optical devices with novel functionalities. In this paper, a new beam-steering system based on double metasurface lenses (metalenses) is proposed and developed. The proposed system is lightweight, small volume, low cost, and easy to integrate. The exact forward and inverse solutions are derived respectively using the generalized Snell's law of refraction. Given the orientations of the double metalenses, the pointing position can be accurately determined. If the desired pointing position is given, the required metalenses' orientations can be obtained by applied global optimization algorithms to solve nonlinear equations related to the inverse problem. The relationships of the scan region and blind zone with the system parameters are derived. The method to eliminate the blind zone is given. Comparison with double Risley-prism systems is also conducted. This work provides a new approach to control light beams.
\end{abstract}

\section{Introduction}

Steering light beams is very important and has found many applications in various optical systems, such as laser scanners, laser communications, laser radar, and other optical systems ${ }^{1-3}$. The common solutions to control light beams include using arrangements of galvo mirrors or pairs of rotatable Risley prisms. Risley prism systems (RPSs) are simple and low cost while particularly effective in continuously scanning the light beams; they have attracted much attention from researchers ${ }^{1-12}$ in the past several decades. Advantages of RPSs include compact size, stability, high precision and resolution, high reliability, etc. Different mathematical models on the forward and inverse formulas $^{4-12}$ with different prism configurations have been implemented to provide scan patterns for the control of light beams. All these conventional methods are based on the classic refraction theorem ${ }^{13}$, namely Snell's law.

Metamaterials, artificial materials capable of providing extraordinary electromagnetic responses not found in nature, have been the hot research topic ${ }^{14,15}$ in the past two decades. Especially in the past decade, considerable efforts ${ }^{16-19}$ have been devoted to the study of metasurfaces. Metasurfaces are two-dimensional (2D) metamaterials that feature subwavelength thickness, low loss, and easy fabrication, promising potential new applications. For the three-dimensional (3D) metamaterials, electrically, we are concerned about effective permittivity, permeability, and refractive index. For metasurfaces, we pay more attention to interface reflection and transmission, including their amplitude, phase, and polarization states. Abrupt phase changes can be introduced on ultrathin metasurfaces, leading to anomalous reflections and refractions that can be explained by generalized laws of reflection and refraction or called generalized Snell's law ${ }^{16}$. They provide an alternative and efficient way to realize intriguing electromagnetic phenomena and devices, such as photonic spin Hall effect $^{20}$, planar holograms ${ }^{21}$, polarization converters ${ }^{17,}{ }^{22}$, anomalous beam generators ${ }^{16,23}$, focusing lenses ${ }^{24,25}$. Optical metasurfaces based on metallic nanostructures ${ }^{26}$, dielectric scatters ${ }^{27}$, and 2D materials such as graphene ${ }^{28}$ are also implemented to modulate the light beams. The new optical lens built on the metasurface platform, namely metalens, have become recent research hotspots for potential multifunctional applications.

In this paper, we develop a light beam scanner using two metasurface lenses (metalens). We apply gradient phases to metalens to redirect the impinging light beams. We use the generalized law of refraction to derive an 
accurate analytical relation between the pointing position and the orientations of the double metalens. We derive the inverse solution that allows finding the desired metalens' orientations at any given pointing position.

The paper is organized as follows: Section 2 derives the exact analytic forward solution of the double-metalens scanner system. It also discusses the scanning accuracy, the scanning range, and the method to eliminate the scan blind zone. Section 3 develops an exact inverse solution. Section 4 concludes.

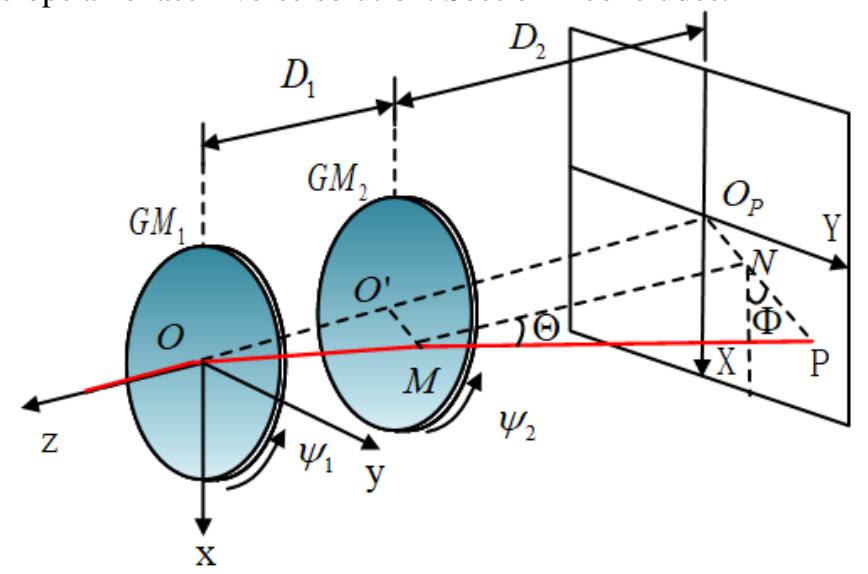

Figure 1. Schematic diagram of the proposed beam-steering double-metalens system.

\section{THE PROPOSED METALENS SCANNER}

Analysis. The schematic of the proposed double-metalens scanner system under the Cartesian coordinate system is shown in Figure 1. It consists of two metalenses $\left(G M_{1}\right.$ and $\left.G M_{2}\right)$ arranged along the z-axis, each of which has a planar structure, a subwavelength thickness, and a pre-determined phase gradient $\left(G_{1}\right.$ or $\left.G_{2}\right)$. The two metalenses can rotate around the z-axis independently. The parameters $\psi_{1}$ and $\psi_{2}$ represent the counterclockwise rotation angles of $G M_{1}$ and $G M_{2}$, respectively, with respect to the z-axis. The distances between the two metalenses and the second metalens and the receiving screen are D1 and D2, respectively. The incident light beam is supposed to be along the $-\mathrm{z}$ axis, passes Center $\mathrm{O}$ of $G M_{1}$ and Point $\mathrm{M}$ of $G M_{2}$, respectively, and finally arrives at Point $\mathrm{P}$ on the receiving screen. The Point $\mathrm{M}$ on $G M_{2}$ can be positioned with the longitudinal and azimuthal angles $(\theta, \varphi)$ with respect to Center $\mathrm{O}$ of $G M_{1}$. The position of Point $\mathrm{P}$ is determined by the coordinates $(\mathrm{X}, \mathrm{Y})$ on the receiving screen and the longitudinal and azimuthal angles $(\Theta, \Phi)$ with respect to Point $\mathrm{M}$ of $G M_{2}$, respectively.

The relationship between the beam direction and the metalenses' orientations is established based on the generalized Snell's law ${ }^{16}$. In the initial state, the phase gradients $G_{1}$ and $G_{2}$ are along the x-axis, namely $\psi_{1}=\psi_{2}=$ 0 . The forward solution is to find out the beam pointing direction or the $(\mathrm{X}, \mathrm{Y})$ on the receiving screen with a set of given metalenses' orientations. Assume $\psi_{1}$ and $\psi_{2}$ is known, the exact formulas can be derived using the generalized Snell's law ${ }^{16}$. First, let us calculate the beam direction of the first metalens $G M_{1}$. The schematic for the 2D generalized Snell's law of refraction is depicted in Figure 2(a). Based on the law, we have

$$
n_{t} \sin \theta_{t}-n_{i} \sin \theta_{i}=\frac{\lambda_{0}}{2 \pi} \frac{d \phi}{d x}, \#(1)
$$

where $n_{t}$ and $n_{i}$ are the refractive index of the relative media, $\lambda_{0}$ is the wavelength of the incident wave, and $\frac{d \phi}{d x}$ is the phase gradient $G_{1}$, along $\mathrm{x}$ axis at the initial time.

As the beam is incident normally on $G M_{1}$, the direction of the leaving or transmitted beam can be expressed as:

$$
\left\{\begin{array}{c}
\theta_{1 t}=\arcsin \left[\left(\frac{G_{1}}{k_{0}}+n_{i} \sin \theta_{1 i}\right) \frac{1}{n_{t}}\right], \#(2) \\
\varphi_{1 t}=\psi_{1}
\end{array}\right.
$$

where $k_{0}=\frac{2 \pi}{\lambda_{0}}$ is the wavenumber. The metalenses are extraordinarily thin, and the media are air (or $n_{i}=1$ and $\left.n_{t}=1\right)$. The pointing direction $(\theta, \varphi)$ of the beam leaving $G M_{1}$ can be found as: 


$$
\left\{\begin{array}{c}
\theta=\theta_{1 t}=\arcsin \left(\frac{G_{1}}{k_{0}}\right) \\
\varphi=\varphi_{1 t}=\psi_{1}
\end{array}\right.
$$

Next, we derive the beam direction $(\Theta, \Phi)$ of $G M_{2}$. From (3), the coordinates of the intersection point $\mathrm{M}$ on the second metalens is $\left(D_{1} \tan \theta_{1 t} \cos \varphi_{1 t}, D_{1} \tan \theta_{1 t} \sin \varphi_{1 t},-D_{1}\right)$.

For the convenience of the derivation, a new coordinate system is set up. It has the origin at Point $\mathrm{M}$ and is rotated by an angle $\psi_{1}$ along the z-axis of the original coordinate system. The relative rotation angle of $G M_{2}$ in the new system is $\psi_{2}-\psi_{1}$ because $G M_{2}$ rotates by an angle of $\psi_{2}$ with respect to the original system. The rotation matrix on the new coordinates, denoted by $\left(x^{\prime}, y^{\prime}, z^{\prime}\right)$, is then given by:

$$
\left[\begin{array}{l}
x^{\prime} \\
y^{\prime} \\
z^{\prime}
\end{array}\right]=\left[\begin{array}{ccc}
\cos \left(\psi_{2}-\psi_{1}\right) & -\sin \left(\psi_{2}-\psi_{1}\right) & 0 \\
\sin \left(\psi_{2}-\psi_{1}\right) & \cos \left(\psi_{2}-\psi_{1}\right) & 0 \\
0 & 0 & 1
\end{array}\right] \times\left[\begin{array}{c}
x \\
y \\
z-D_{1}
\end{array}\right] . \#(4)
$$

With the phase gradient $G_{2}$ initially along the x-axis on $G M_{2}$, corresponding to $\vec{G}_{2}=\left(G_{2}, 0,0\right)^{T}$, the rotated phase gradient vector is given by

$$
\vec{G}_{2}^{\prime}=\left(G_{x}, G_{y}, G_{z}\right)=\left[G_{2} \cos \left(\psi_{2}-\psi_{1}\right), G_{2} \sin \left(\psi_{2}-\psi_{1}\right), 0\right] . \#(5)
$$

With the 3D generalized Snell's law, whose schematic is shown in Figure 2(b), the following formula is obtained

$$
\left\{\begin{array}{c}
\cos \theta_{2 t} \sin \varphi_{2 t}=\frac{G_{y}}{n_{2 t} k_{0}} \\
n_{2 t} \sin \theta_{2 t}-n_{2 i} \sin \theta_{2 i}=\frac{G_{x}}{k_{0}}
\end{array}\right.
$$

With $n_{2 i}=n_{2 t}=1$ and $\theta_{2 i}=\theta,\left(\theta_{2 \mathrm{t}}, \varphi_{2 \mathrm{t}}\right)$ can be derived from (6)

$$
\left\{\begin{array}{c}
\theta_{2 t}=\arcsin \left[\frac{G_{1}}{k_{0}}+\frac{G_{2} \cos \left(\psi_{2}-\psi_{1}\right)}{k_{0}}\right] \\
\varphi_{2 t}=\arcsin \left[\frac{G_{2} \sin \left(\psi_{2}-\psi_{1}\right)}{k_{0} \cos \theta_{2 t}}\right]
\end{array}\right.
$$

Then by transforming $\left(\theta_{2 t}, \varphi_{2 t}\right)$ into the spherical coordinate $(\theta 2, \varphi 2)$ on the new coordinate system, we have

$$
\left\{\begin{array}{l}
\theta_{2}=\arccos \left(\cos \theta_{2 t} \cos \varphi_{2 t}\right) \\
\varphi_{2}=\arcsin \left(\frac{\cos \theta_{2 t} \sin \varphi_{2 t}}{\sin \theta_{2}}\right)
\end{array}\right.
$$

By rotating the new coordinate system back to its original, we have:

$$
(\Theta, \Phi)=\left(\theta_{2}, \varphi_{2}+\psi_{1}\right) \text { \#(9) }
$$

The coordinate $(\mathrm{X}, \mathrm{Y})$ of Point $\mathrm{P}$ on the receiving screen is obtained by

$$
\left\{\begin{array}{c}
X=D_{1} \tan \theta \cos \varphi+D_{2} \tan \Theta \cos \Phi \\
Y=D_{1} \tan \theta \sin \varphi+D_{2} \tan \Theta \sin \Phi
\end{array}\right.
$$




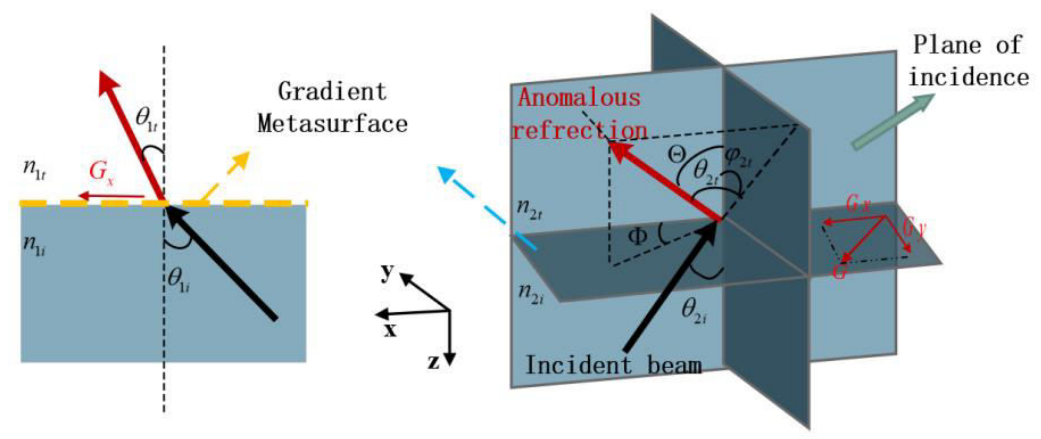

(a)

(b)

Figure 2. (a) 2D and (b) 3D generalized Snell's law of refraction

The Scan Blind Zone. A scan blind zone occurs when the phase gradients of the two metalenses are identical, leading to the target lost within the scan region. Here an example is given to illustrate and discuss the problem. With $G_{1}=G_{2}=4188.8 \mathrm{rad} / \mathrm{mm}, D_{1}=10 \mathrm{~mm}, D_{2}=20 \mathrm{~mm}$, and $\lambda_{0}=500 \mathrm{~nm}$ (corresponding to $k_{0}=0.0126 \mathrm{~nm}^{-1}$ ), the scan region on the receiving screen is calculated and plotted in Figure 3(a). There is a circular blind zone in the center of the circular scan region. Based on the forward solution above, the blind zone is determined by the phase gradient and occurs when the gradients of $\mathrm{GM}_{1}$ and $\mathrm{GM}_{2}$ are identical. Based on the geometrical relationship between the leaving beam of $\mathrm{GM}_{1}$ and the intersection point $\mathrm{M}$, the radius of the blind zone, $\left|\mathrm{O}^{\prime} \mathrm{M}\right|$, is:

$$
\left|O^{\prime} M\right|=D_{1} \tan \left(\theta_{1 t}\right)=D_{1} \tan (\theta) \text {.\#(11) }
$$

Since the altitude angle $\theta$ increases with the phase gradient G1, the blind zone will increase with $D_{1}$ and $G_{1}$.

Increasing the phase gradient $G_{2}$ of $\mathrm{GM}_{2}$ is a solution to decrease or eliminate the scan blind zone. From (7) and (8), when $\left|\psi_{2}-\psi_{1}\right|=180^{\circ}$, we have:

$$
\theta_{2}=\theta_{2 \mathrm{t}}=\arcsin \left[\frac{G_{1}}{k_{0}}-\frac{G_{2}}{k_{0}}\right]
$$

With the geometrical relationship between the emerging beam of $\mathrm{GM}_{2}$ and the scan point $\mathrm{P}$ on the receiving screen, we get:

$$
|N P|=D_{2} \tan (\theta)
$$

Combining (11), (12), and (13), we have the increment of $G_{2}$ :

$$
\Delta \mathrm{G}=\left|G_{2}-G_{1}\right|=k_{0} \sin \left(\operatorname{atan}\left(\frac{\left[O^{\prime} M\right]}{D_{2}}\right)\right) . \#(14)
$$

Figure 3(b) plots the scan region without a blind zone, where the increment of $G_{2}$ is $2187.5 \mathrm{rad} / \mathrm{mm}$, corresponding to an enlarged phase gradient $\mathrm{G}_{2}^{\prime}=6376.3 \mathrm{rad} / \mathrm{mm}$.

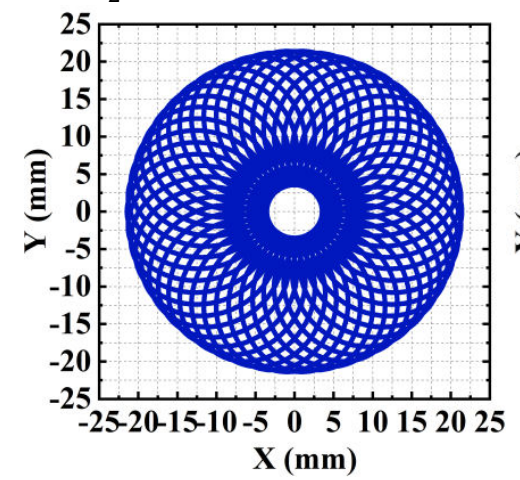

(a)

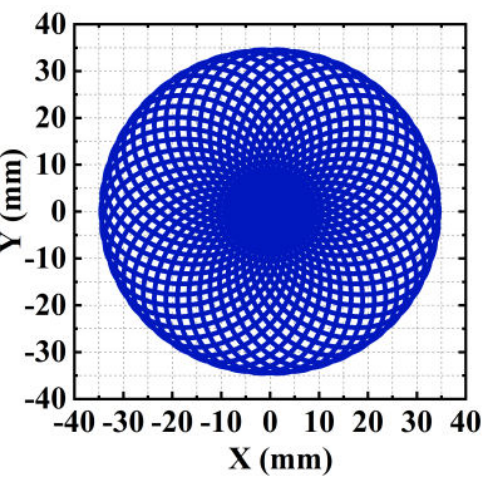

(b)

Figure 3. The beam scan regions (a) with the blind zone and (b) with the beam blind zone eliminated. 
The Scan Region. In Figure 3, not only the blind zone is eliminated, but also the scan region enlarged when the phase gradient $G_{2}$ increases. Therefore, it is necessary to discuss the impact of phase gradients on the scan region. The maximum $X$ and $Y$ of the scan region on the receiving screen can be obtained when $\left|\psi_{2}-\psi_{1}\right|=0^{\circ}$. From (10), we have:

$$
X_{\text {max }}=Y_{\text {max }}=D_{1} \tan \left[\arcsin \left(\frac{G_{1}}{k_{0}}\right)\right]+D_{2} \tan \left[\arcsin \left(\frac{G_{1}}{k_{0}}+\frac{G_{2}}{k_{0}}\right)\right] . \#(15)
$$

Next, we show how the scan region varies with the parameters of the double-metalens system. For the convenience of the analysis, the phase gradients of $\mathrm{GM}_{1}$ and $\mathrm{GM}_{2}$ are set to be identical $\left(G=G_{1}=G_{2}\right)$. Figure 4 illustrates the relations among (Xmax, Ymax), $D_{1} / \lambda_{0}, D_{2}$, and $G / k_{0}$. It is obvious that the scan region will increase with $D_{1} / \lambda_{0}, D_{2}$, and $G / k_{0}$.

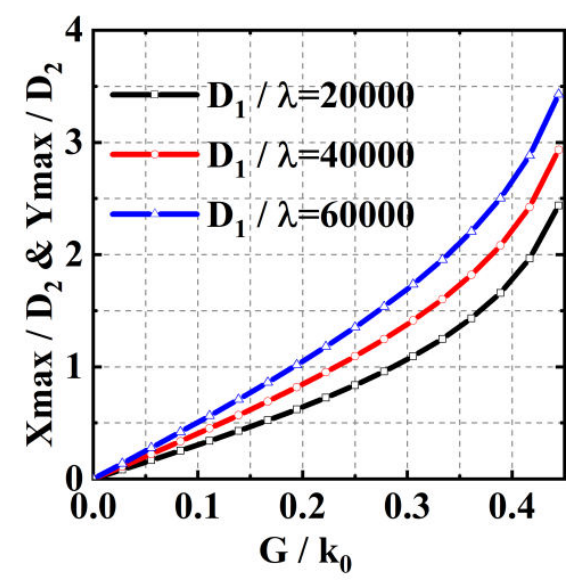

Figure 4. Influence of $D_{1}, D_{2}$, and $G / k_{0}$ on the beam scan region defined by $\left(X_{\max }, Y_{\max }\right)$.

Impacts of the Distance Between Metalenses. The distance between metalenses could not be too large. If the two metalenses are placed too far away from each other, the emerging beam from $G M_{1}$ may propagate beyond the aperture of $G M_{2}$. For example, if two identical metalenses are taken with aperture $D=200 \mathrm{~mm}$ and $\frac{G_{1}}{k_{0}}=0.33$, the elevation angle $\theta_{1 t}$ is $19.47^{\circ}$ and the maximum distance can be calculated below:

$$
D_{1 \text { max }}=\frac{D / 2}{\tan \left(\theta_{1 t}\right)}=282.8 \mathrm{~mm} \text {. \#(16) }
$$

In practice, a smaller distance is preferred to reduce the scan blind zone ${ }^{29}$. However, if the distance between $G M_{1}$ and $G M_{2}$ is too close, mutual coupling between metalenses may become unacceptly large. Hence the compromised distance value is taken. In addition, the minimum distance is dependent on the specific characteristics of the metelenses, while the maximum distance on the wavelength of the incident beam, the size of the lens aperture, and the phase gradient of the metalenses.

The distance $D_{1}$ also impacts the beam trajectories ${ }^{11,29}$ because it affects the scan region. Figure 5 illustrates the differences in trajectories when $D_{2}$ is constant. It is seen that the impact of $D_{1}$ can be ignored when $D_{2} / D_{1}$ ratio is larger than 40. 


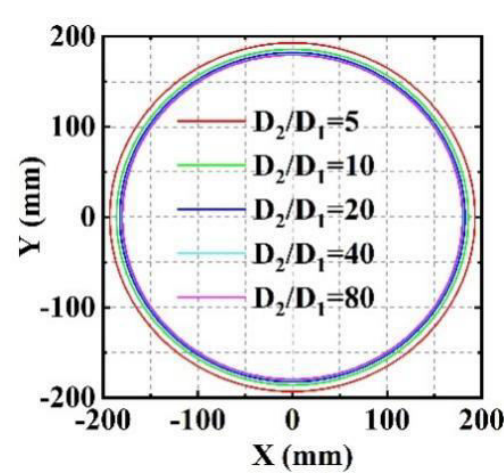

(a)

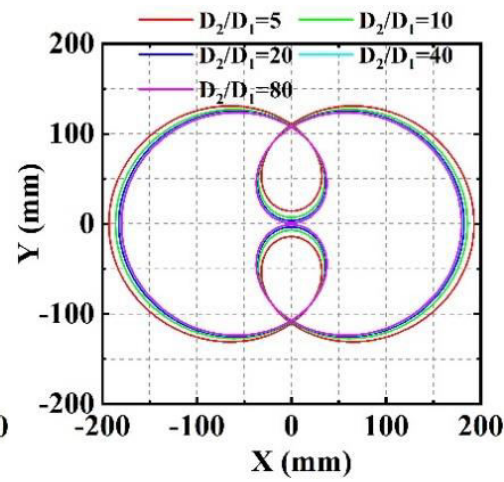

(b)

Figure 5. Beam trajectories with different $D_{2} / D_{1}$ ratios (a) $\omega_{1}=\omega_{2}$ (b) $\omega_{1}=3 \omega_{2}$, where $\omega_{1}$ and $\omega_{2}$ are the rotationally angular speed of the two metalens ${ }^{11,29}$.

Comparison with the Paraxial Approximation Solution of Risley-Prism Systems. In the conventional double Risley-prism systems, the first-order paraxial method ${ }^{7,29}$ is a typical solution for RPSs with thin prisms that have small deviation angles. However, since the first-order approximation is applied, it only works when the approximation is good enough. Next, comparisons with the first-order method ${ }^{7,29}$ with RPSs are conducted to demonstrate the accuracy of the proposed method. The mathematical expressions of the first-order method expressions are:

$$
\left\{\begin{array}{c}
\Theta=\sqrt{\delta_{1}^{2}+\delta_{2}^{2}+2 \delta_{1} \delta_{2} \cos \left(\psi_{1}-\psi_{2}\right)} \\
\Phi=\arctan \left(\frac{\delta_{1} \sin \left(\psi_{1}\right)+\delta_{2} \sin \left(\psi_{2}\right)}{\delta_{1} \cos \left(\psi_{1}\right)+\delta_{2} \cos \left(\psi_{2}\right)}\right)
\end{array}, \#(17)\right.
$$

where $\delta_{1}$ and $\delta_{2}$ denote the deviation angles by the two prisms, respectively. The relationship between the deviation angle and the phase gradient with the proposed double-metalens scanner system can be obtained from (3):

$$
\delta=\arcsin \left(\frac{G}{k_{0}}\right)
$$

In the comparison, the double-metalens scanner system with two identical metalenses is considered. Let $\frac{G_{1}}{k_{0}}$ and $\frac{G_{2}}{k_{0}}$ be 0.25 and 0.33 , respectively, which corresponds to the opening angles of $28.96^{\circ}$ and $38.94^{\circ}$ of the prisms, respectively, the comparisons are made between the first-order method and the proposed analytic solution. The beam trajectory is acquired by keeping $\psi_{1}=90^{\circ}$ and varying $\psi_{2}$ from $0^{\circ}$ to $360^{\circ}$.

Figure 6 shows the two comparison results. When the phase gradient is small, corresponding to the small opening angle of the prism, the two results are relatively close (see Figure 6 (a)). When the phase gradient or the opening angle increases, the differences between the two results increase (see Figure 6 (b)). The reason is that increasing the opening angle of the prism will introduce a larger error due to the first-order approximation ${ }^{7,29}$ while increasing the phase gradient will not change the accuracy of the proposed analytical solution. An increase of the phase gradient of the metalens or the opening angle of the prism in the two systems leads to the increase of the scanning region. Therefore, the proposed metalens system always gives accurate solutions for any pre-determined scanning region without changing the volume of the system. 


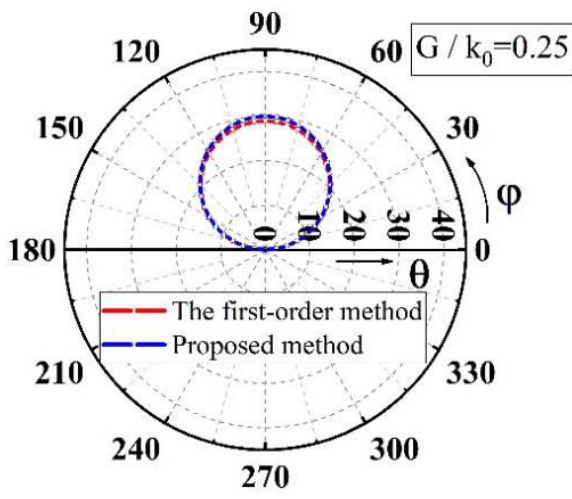

(a)

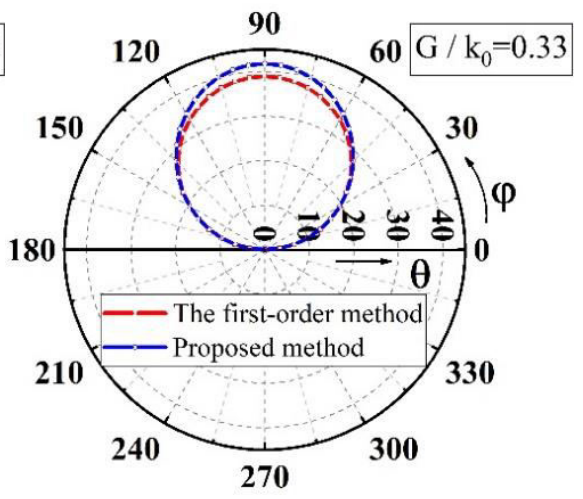

(b)

Figure 6. Comparisons for the beam trajectory when $\psi_{1}=90^{\circ}$ and $\psi_{2}$ varies from $0^{\circ}$ to $360^{\circ}$. (a) $\mathrm{G} / k_{0}=0.25$; (b) $\mathrm{G} / k_{0}=0.33$.

\section{INVERSE SOLUTION AND TARGET TRACKING PERFORMANCE FOR DOUBLEL-METALENS SYSTEMS}

Inverse Solution. The inverse solution is to find out the orientation angles of the metalenses for a given pointing position on the receiving screen. It is especially significant in practical applications such as target tracking. Following the exact derivation of the forward solution of the new double-metalens system, an exact inverse solution is found as follows.

Equation (8) can be rewritten in the following form when substituting (9) into (8):

$$
\left\{\begin{array}{l}
\cos \theta=\cos \theta_{2 t} \cos \varphi_{2 t} \\
\sin \theta=\frac{\cos \theta_{2 t} \sin \varphi_{2 t}}{\sin \left(\varphi-\psi_{1}\right)}
\end{array}\right. \text { \#(19) }
$$

Then (19) can be further derived as:

$$
\begin{gathered}
\tan \theta_{2 t}=\tan \theta \sin \left(\varphi-\psi_{1}\right), \#(20) \\
\cos ^{2} \theta_{2 t}=1-\sin ^{2} \theta \cos ^{2}\left(\varphi-\psi_{1}\right) . \#(21)
\end{gathered}
$$

Let $\mathrm{A}=\frac{G_{1}}{k_{0}}$ and $\mathrm{B}=\frac{G_{2}}{k_{0}}$, the new expressions of (7) are:

$$
\begin{gathered}
\sin \theta_{2 t}=A+B \cos \left(\psi_{2}-\psi_{1}\right), \#(22) \\
\sin \varphi_{2 t} \cos \theta_{2 t}=B \sin \left(\psi_{2}-\psi_{1}\right) . \#(23)
\end{gathered}
$$

Substituting (21) into (22), we get:

$$
\sin \theta \cos \left(\varphi-\psi_{1}\right)=A+B \cos \left(\psi_{2}-\psi_{1}\right) . \#(24)
$$

Solving (8), (9), and (23) simultaneously, we have:

$$
\sin \theta \sin \left(\varphi-\psi_{1}\right)=B \sin \left(\psi_{2}-\psi_{1}\right) . \#(25)
$$

The orientations $\psi_{1}$ and $\psi_{2}$ can be obtained by solving (10), (24), and (25) through optimization algorithms, for example, genetic algorithm, particle swarm optimization algorithm, differential evolution algorithm, etc. In this work, we used the MATLAB function vpasolve to get the optimized solutions.

Target Tracking Performance. An asteroid target trajectory is applied to validate the performance of the target tracking using the proposed inverse solution; the expression of the asteroid is:

$$
\left\{\begin{array}{l}
X=100 \cos ^{3}(\theta) \\
Y=100 \sin ^{3}(\theta)
\end{array}, \quad 0 \leq \theta \leq 2 \pi . \#(26)\right.
$$


The MATLAB function vpasolve is used for the solution. The fitness function is the sum of the absolute value of (10), (24), and (25).

With $A=B=0.33, \mathrm{D}_{1}=20 \mathrm{~mm}$, and $\mathrm{D}_{2}=200 \mathrm{~mm}$, the trajectory and the inverse solutions to $\psi_{1}$ and $\psi_{2}$ are plotted in Figure_7(a) and (b), respectively. Substituting the orientations obtained from the inverse solution into the analytic forward solution, we found that the errors are less than $1 \mu \mathrm{m}$.

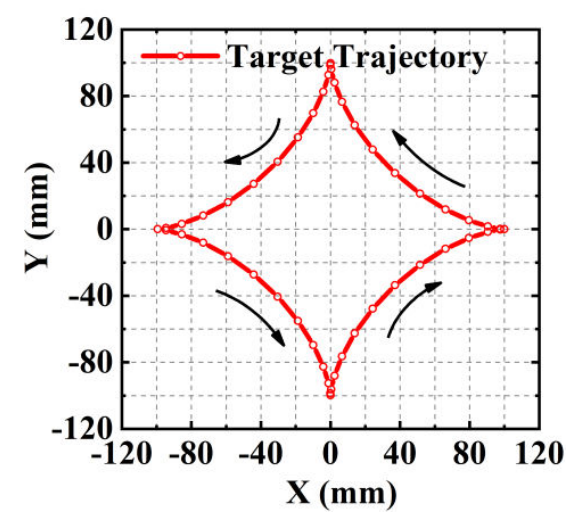

(a)

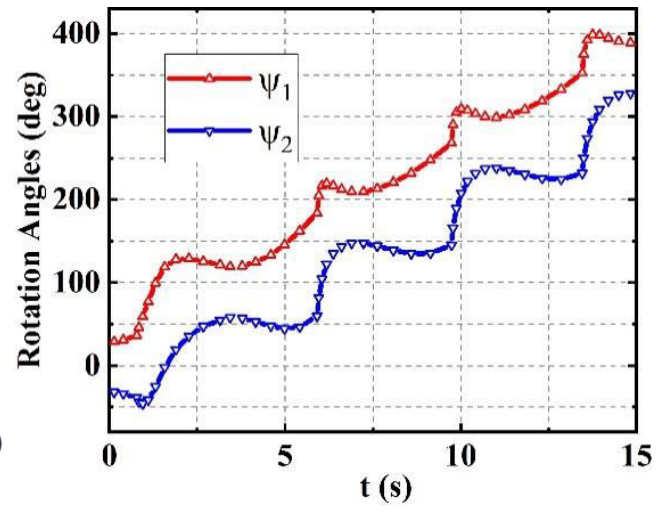

(b)

Figure 7. (a) The given asteroid target trajectory, (b) the inverse solutions to the orientations of $\mathbf{G M}_{\mathbf{1}}$ and $\mathbf{G M}_{\mathbf{2}}$.

\section{DISCUSSION AND CONCLUSIONS}

The optical metalens is currently an important under-explored topic. There are two kinds of metalenses: metallic plasmonic one and all-dielectric one. The latter is free from ohmic losses, leading to a much higher efficiency of operation. The beam-steering systems-based metalenses are lightweight, compact, and easy to be integrated because the volumes of metalenses do not vary much with the change of phase gradients.

The exact forward and inverse solutions for beam-steering systems with double metalenses are successfully derived based on the generalized Snell's law of refraction in this paper. The relationships among the scan region, the blind zone, the beam trajectory, and system parameters are derived or discussed. The approach to eliminating the scan blind zone is presented and numerically demonstrated. Comparison of the new system with the conventional Risley-prism systems is carried out, demonstrating that the proposed method can give effective and accurate results.

There are some limitations with the current double-metalens beam-steering systems. Due to the dispersions of metalenses and the refraction, they are more applicable to monochromatic light beams. The optimization algorithms to solve the inverse solution converge slowly. More efficient methods are needed for practical applications.

\section{References}

1. Rosell, F. A. Prism scanners, J. Opt. Soc. Am. 50, 521-526 (1960).

2. Marshall, G. F. Handbook of Optical and Laser Scanning, 2nd ed. (CRC Press, 2011).

3. Li, A. Double-Prism Multi-mode Scanning: Principles and Technology (Springer Series in Optical Sciences, 2018).

4. Lacoursiere, J. et al. Large deviation achromatic Risley prisms pointing systems. Proc. SPIE 4773, Optical Scanning 2002. 4773, 123-131 (2002).

5. Duncan, B. D., Bos, P. J. \& Sergan, V. Wide-angle achromatic prism beam steering for infrared countermeasure applications. Opt. Eng. 42(4), 1038-1047 (2003).

6. Sun, J., Liu L., Yun, M., Wang, L. \& Zhang, M. The effect of the rotating double-prism wide-angle laser beam scanner on the beam shape. Optik 116, 553-556 (2005). 
7. Yang, Y. Analytic solution of free space optical beam steering using Risley prisms. J. Lightwave Technol. 26(21), 3576-3583 (2008).

8. Li, Y. Closed form analytical inverse solutions for Risley-prism-based beam steering systems in different configurations. Appl. Opt. 50, 4302-4309 (2011).

9. Li, Y. Third-order theory of the Risley-prism-based beam steering system. Appl. Opt. 50, 679-686 (2011).

10. Li, A. Sun, W., Yi, W. \& Zuo, Q. Investigation of beam steering performances in rotation Risley-prism scanner. Opt. Express 24(12), 12840-12850 (2016).

11. Li, A., Liu, X. \& Sun, W. Forward and inverse solutions for three-element Risley prism beam scanners. Opt. Express 25(7), 7677-7688 (2017).

12. Bawart, M., Bregenzer, N., Bernet, S. \& Ritsch-Marte, M. Dynamic beam-steering by a pair of rotating diffractive elements. Opt. Comm. 460, 125071 (2020).

13. Jenkins, F. R. \& White, H. E. Fundamentals of Optics, 4th ed. (McGraw-Hill, 2001)

14. Engheta, N. \& Ziolkowski, R. W. Metamaterials: Physics and Engineering Explorations, (Wiley-IEEE Press, 2006)

15. Cai, W. \& Shalav, V. Optical Metamaterials: Fundationals and Applications, (Heidelberg: Springer-Verlag, 2010)

16. Yu, N. et al. Light Propagation with Phase Discontinuities: Generalized Laws of Reflection and Refraction, Science 334, 333 (2011).

17. Li, Z. C., Liu, W. W., Cheng, H., Chen, S. Q. \& Tian, J. G. Realizing Broadband and Invertible Linear-tocircular Polarization Converter with Ultrathin Single-layer Metasurface. Sci Rep-UK 5, 6328-6333, (2015).

18. Holloway, C. L. et al. An Overview of the Theory and Applications of Metasurfaces: The Two-Dimensional Equivalents of Metamaterials, IEEE Antennas Propag. Mag. 54, 10 (2012).

19. Wan, X., Jia, S. L., Cui, T. J. \& Zhao, Y. J. Independent modulations of the transmission amplitudes and phases by using Huygens metasurfaces. Sci Rep-UK 6, 25639, (2016).

20. Yin, X. B., Ye, Z. L., Rho, J., Wang, Y. \& Zhang, X. Photonic Spin Hall Effect at Metasurfaces. Science 339, 1405-1407, (2013).

21. Ni, X. J., Kildishev, A. V. \& Shalaev, V. M. Metasurface holograms for visible light. Nat Commun 4, 2087, (2013).

22. Yu, N. et al. A Broadband, Background-Free Quarter-Wave Plate Based on Plasmonic Metasurfaces. Nano Lett 12, 6328-6333, (2012).

23. Sun, S., Yang, K. Y., Zhou, L. \& Tsai, D. P. High-Efficiency Broadband Anomalous Reflection by Gradient Meta-Surfaces. Nano Lett 12, 6223-6229, (2012).

24. Aieta, F. et al. Aberration-Free Ultrathin Flat Lenses and Axicons at Telecom Wavelengths Based on Plasmonic Metasurfaces. Nano Lett 12, 4932-4936, (2012).

25. Wang, P., Mohammad, N. \& Menon, R. Chromatic-aberration-corrected diffractive lenses for ultra-broadband focusing. Sci Rep-UK 6, 21545, (2016).

26. Yu, P. et al. Controllable optical activity with non-chiral plasmonic metasurfaces", Light-Sci Appl. 5, e16096, (2016)

27. Zhan, A., Gibson, R., Whitehead, J., Smith, E., Hendrickson, J. R.\& Majumd, A. Controlling three-dimensional optical fields via inverse Mie scattering. Sci Adv. 5, eaax4769, (2019)

28. Cheng, H. et al. Dynamically tunable broadband infrared anomalous refraction based on graphene metasurfaces. Adv Opt Mater. 3, 1744-1749 (2015)

29. Lu, Y., Zhou, Y., Hei, M. \& Fan, D. Theoretical and experimental determination of steering mechanism for Risley prism systems. Appl. Opt. 52(7), 1389-1398 (2013) 


\section{Acknowledgements}

This work was supported in part from the National Science Foundation of China (NSFC) (62071187, 62071125), and in part from the Natural Science Foundation of Fujian Province (2018J01805, 2021J01581, 2021J01288).

\section{Author contributions statement}

J.R.W and Y.H.G. derived the theoretical solutions; Y.H.G conceived the idea; J.R.W did the theoretical calculations; Z.M.X and H.Z. analyzed the results; J.R.W prepared all figures; J.R.W, Y.H.G and Z.Z.C wrote the manuscript with the help of Z.M.X and H.Z.; All authors reviewed the manuscript; Y.H.G. supervised the project.

\section{Additional information}

The authors declare no competing financial interest. 